\title{
ELECTRONIC TRANSPORT AND LASING IN MICROSTRUCTURES
}

\author{
PROGRESS REPORT
}

For the Period November 1, 1991 to October 31,1992

\author{
Prepared for \\ THE U. S. DEPARTMENT OF ENERGY \\ DE $-F G 02-84$ ER 45058
}

\section{DISCLAIMER}

This report was prepared as an account of work sponsored by an agency of the United States Government. Neither the United States Government nor any agency therenf, nor any of their employees, makes any warranty, express or implied, or assumes any legal liability or tesponsibility for the accuracy, completeness, or usefulness of any information, apparatus, product, or process disclosed. or represents that its use would not infringe privately owrted rights. Reference herein to any specific commercial product, process, or service by trade name. trademark, manufacturer, or otherwise does not necessarily constitute or imply its endorsement, recom. mendation, or favoring by the United States Government or any agency thereof. The views and opinions of authors expressed herein do not necessarily state or reflect those of the United States Government or any agency theroof.

Prepared by:

Professor Melvin Lax

Principal Investigator

Department of Physics

The city college of CUNY

Convent Avenue a 138 th street

New York, NY 10031

\section{MASTER}




\title{
Electronic Transport and Lasing in Microstructures PROGRESS REPORT
}

\author{
Prepared for \\ The U.S. Department of Energy \\ DE-FG(02-84ER45058 \\ Principal Investigator: Melvin Lax
}

Physics Department, City College of the City University of New York

\section{Brief Summary of Progress}

We consider the interaction of hot carriers with hot phonons in a quantum well. Transport is considered in the transverse direction and turneling through the well barriers. Time-dependent transport effects down to the femto-second regime are included, as are strong and/or microwave fields. with negative resistance effects. Resonant tunneling assisted by phonon relaxation and infra-red radiation will be explored. The limitations on transmission of information due to partition noise, as influenced by the design of semiconductor feedback lasers will be considered. The use of light scattering and decision theory to detect shell-like aerosols is examined. 


\section{SUMMARY OF ACCOMPLISHMENTS}

\section{Inelastic Electron Tunneling in Devices With Spatial Structure}

Tunneling through double barriers or heterosiructures is important in electronics. We have recently proposed a solvable model based on space independent interactions, ${ }^{1,2}$ and at Green's function method that permits space dependence. ${ }^{3}$ Arbitrary barrier structures are permitted. Interaction with optical phonons and infra-red photons has been shown to lead to a strong enhancement of tunneling. 1,3

Applying our approach, we have calculated the high-frequency current through a double-barrier tunneling structure. In contrast to previous results, we point out that the highfrequency current has a wave behavior and this behavior will affect experimental measurements of the high-frequency current in tunneling devices. This result published by us $^{4}$ has been noted and referred to by several groups, including the IBM research center and others.

Recently, we have developed an approach in study of the time response of the transmitted current through a double-barrier turneling structure to an applied pulse. Due to the difficulty of the problem, previous calculations, using the Wigner function method, were performed on supercomputers. We use a Green's function approach developed by us, that is more efficient. We have obtained quantitatively correct results using only a workstation. This paper is in preparation.

\section{Non-perturbative approach to electron tunneling with dissipation}

Recently, we developed an approach to study the intluence of the environment on electron tunneling. We have proven that our approach has the same influence functional as the one given by Feynman and Vernon ${ }^{5}$. The tunneling rate of an electron coupled with phonons is derived nonperturbatively using the WKB method. The tunneling asymptotic behavior at low and high temperature limits are obtained ${ }^{6}$. 


\section{Transport in Quasi-2 Dimensional Systems}

We have developed a new approach to handle high-frequency conductivity in the presence of a strong electric field. ${ }^{7}$ This approach includes the memory effect and is able to compute high-harmonic components of current and electron temperature.

We have shown that the life time of photo-excited electrons in a $\Gamma-X$ system could be much longer than that in the pure $\Gamma$ system. ${ }^{8}$

\section{Quasi-Binary Decision Making Using Light Scattering}

The ability to distinguish between homogeneous aerosols, such as water droplets, and encapsulated droplets which could possess bacteriological contents is of some importance. This raises interesting basic problems. Can one, with a small number of light scattering measurements, solve the inverse problem of the possible radial dependence of the index of refraction?

Such inverse problems are known to be ill-posed ${ }^{9}$ in the presence of noise. They can not be solved without some regularization that restricts the possible solutions. ${ }^{10}$ In our case, we regularize the problem by requiring that we have only a homogeneous sphere, or a shell with an index different from that of the interior.

Since noise at the $10 \%$ level is present. and a wide range of outer radii (saly from . $1 \mu$ to $10 \mu$ ) is possible the problem appears hopeless even with a reasonable range of indices of refraction for the sphere and shell. With a limited number of measured scattered intensities, It would seem possible to represent most data as possibly coming from either kind of scatterer. Moreover, our problem is a mixed detection and estimation problem. One can not make the appropriate binary decision without also estimating the best possible choices of indices and radii. Thus some old techniques of decision theory due to Bayes, and Neyman and Pearson ${ }^{11,12}$, must be combined with estimation theory to make a combined analysis. In the language of communication theory, an estimation problem must the combined with a detection problem. ${ }^{13,14}$

Instead of making a frontal attack on the problem, containing two parameters for the sphere (radius and index) and four parameters for the shell (two radii and two indices), we started by considering restricted cases: 
1. A single free parameter for the sphere and one for the shell

2. Two parameters for the sphere and two for the shell.

3. Two free parameters for the sphere and three for the shell.

4. Two parameters for the sphere and four for the shell.

Since our results are not readily available, tive viewgraphs are enclosed. The first viewgraph summarizes the parameters for the runs in the later viewgraphs. These viewgraphs were made by a Monte Carlo calculation in which a range of index and radius parameters are used and a comparison is made between theoretical Mie scattering for a sphere (or a shell) is made with pseudo experimental results for a thousand cases of each kind. The latter are made by selecting parameters at random and also adding $5 \%$ or $10 \%$ noise. The parameter $v_{1}$ is obtained for each experimental particle by making a least squares fit with spherical scatterers over the given range of index and radius. The parameter $v_{2}$ is obtained by a least square fit over the domain os shell parameters.

When $\log v_{1} / v_{2}$ is negative, the fit with a sphere is better than that with a shell. The two figures of each plot were made using spherical and shell scattering respectively. Viewgraph 2 shows a small overlap between the two figures indicating that it would be easy to distinguish between these cases. But Viewgraph 2 was made with a narrow range of permitted parameters. Viewgraph 3, made with a wider range shows overlap, and difficulty in deciding between the cases. This demonstrates that the use of only 5 measurements of intensity are inadequate for this case.

We next did a two parameter-two parameter calculation but omit the results here. We were encouraged by these results to try a two parameter sphere and a three parameter shell. (This was accomplished by restricting the outer shell radius to equal that of the sphere.) These results also show an excellent separation. See Viewgraph 4. But this was accomplished by using 12 measurements (two polarizations and six angles).

The difficulty associated with these calculations are shown in Viewgraph 5 which displays the function $v_{1}$ which was minimized to get the best fit for the sphere. The numerous minima are associated with the large size parameters permitted for the sphere in this case, as described in Viewgraph 1. 


\section{RANGES OF PARAMETERS}

Wavelength $=.4416 \mu$

\section{One Parameter, $5 \%$ Noise, 5 Measurements}

Sphere with radius $R=2.5 \mu$ and $1.33<m<1.55$

Shell with radius $R_{\text {out }}=2.5 \mu$ and $1.76 \mu<R_{\text {in }}<2.2 \mu$ and fixed $m_{\text {in }}=1.33, m_{\text {out }}=1.55$

One Parameter (Wider range), 5\% Noise, 5 Measurements

Sphere with radius $0.1 \mu<R<10 \mu$

Shell with radius $0.1 \mu<R_{\text {out }}<10 \mu$ and $R_{\text {in }}=0.9 R_{\text {out }}$ (size parameter $x=2 \pi R_{\text {out }} \lambda, 1.42<x<142.28$ )

Two Parameter Sphere and Three Parameter Shell $10 \%$ Noise, 12 Measurements

Sphere with parameters $4<R \wedge<8$ and $1.33<m<1.8$

Shell with parameter $4 \leq R_{\text {in }} \wedge \leq 6, R_{\text {out }} \wedge=8$,

$1.33 \leq m_{\text {in }} \leq 1.55$ and $1.6 \leq m_{\text {out }} \leq 1.8$ 


\section{ONE PARAMETER FIT}

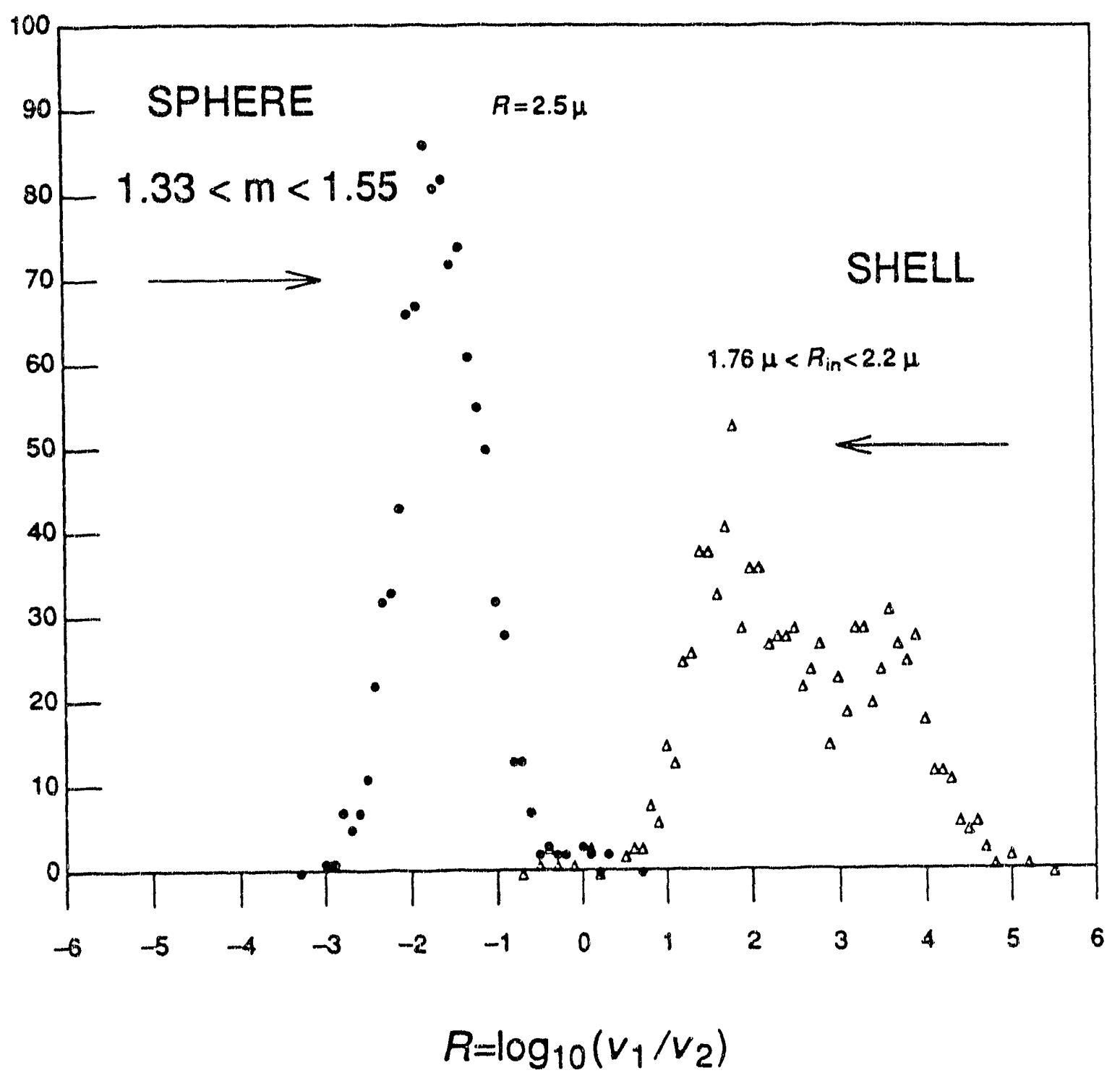

1000 tries.

Data at $\theta=45,50,55,60,65$. 


\section{ONE PARAMETER FIT (WIDER RANGE)}

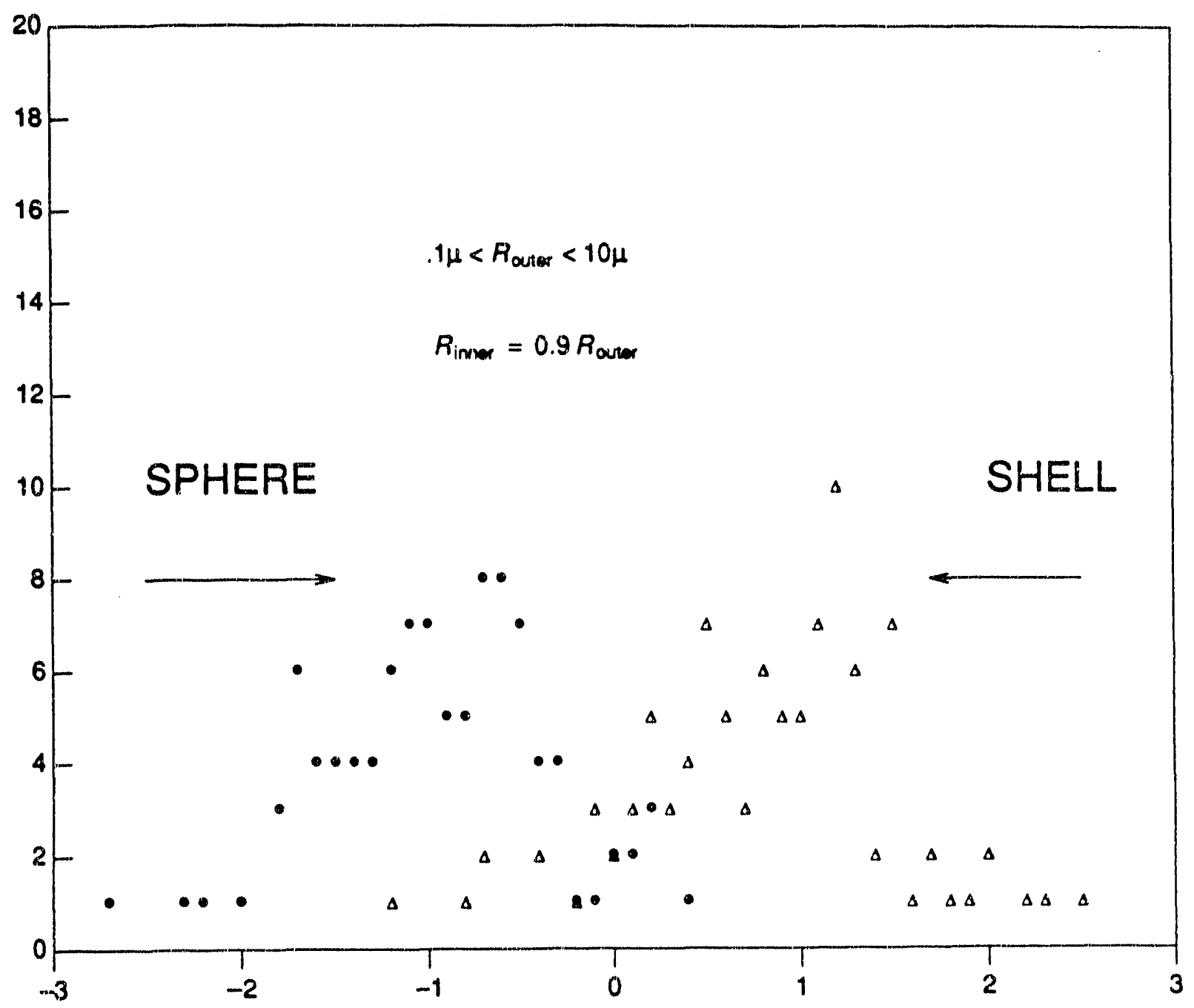

$\log _{10}\left(v_{1} / v_{2}\right)$

100 tries. Data at $\theta=45,50,55,60,65$. 


\section{TWO-THREE PARAMETER FIT}

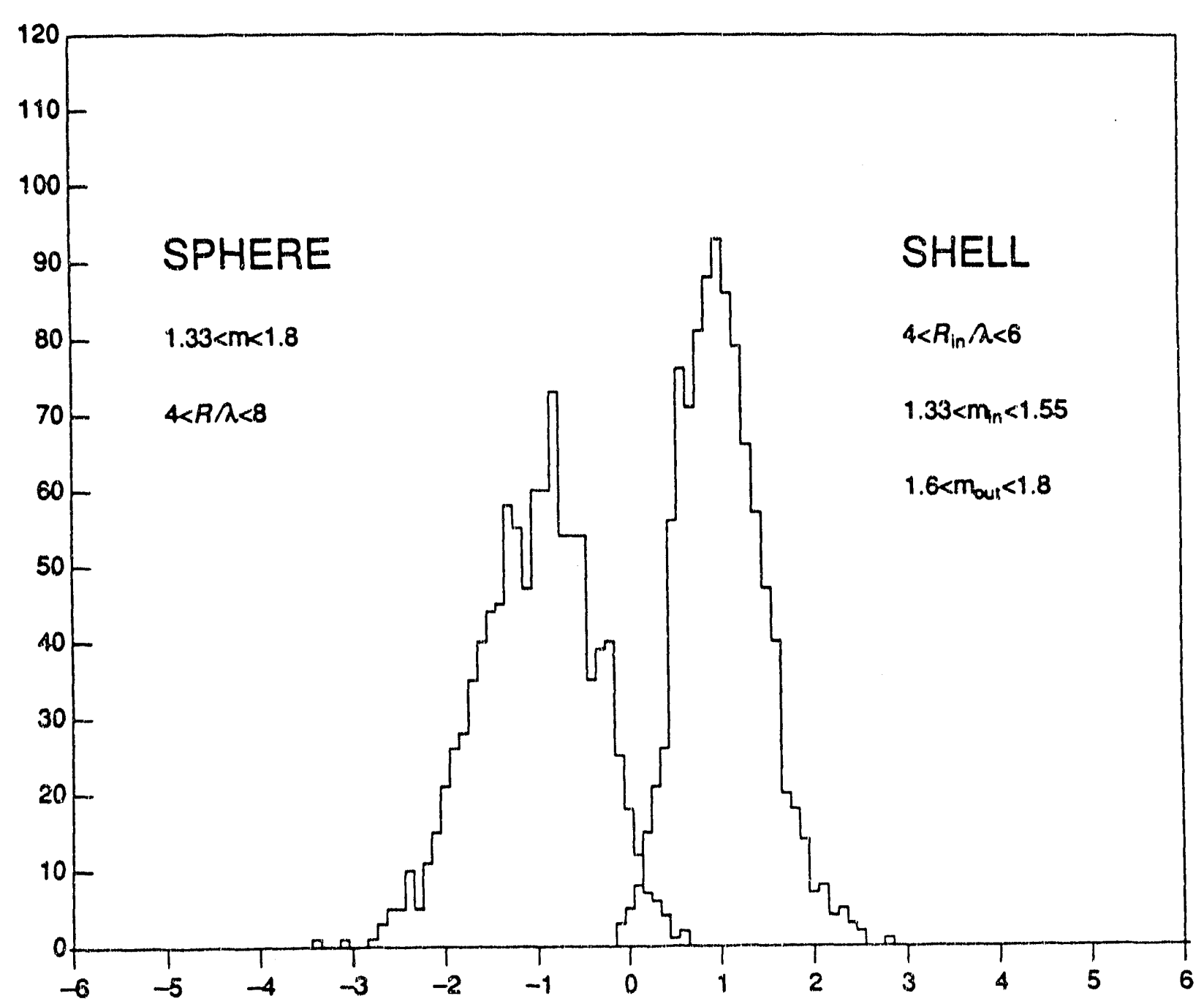

$\log _{10}\left(v_{1} / v_{2}\right)$

1000 tries.

$H$ and $V$ polarized detection at $\theta=90,105,120,135,150,165$. 


\section{MINIMIZATION PROFILE FOR SPHERE Two Parameters}

$$
v\left(\eta^{(1)}\right)=\sum_{i}\left[l_{\exp }\left(\theta_{i}, \eta^{*}\right)-l\left(\theta_{i}, \eta^{(1)}\right)\right]^{2}
$$

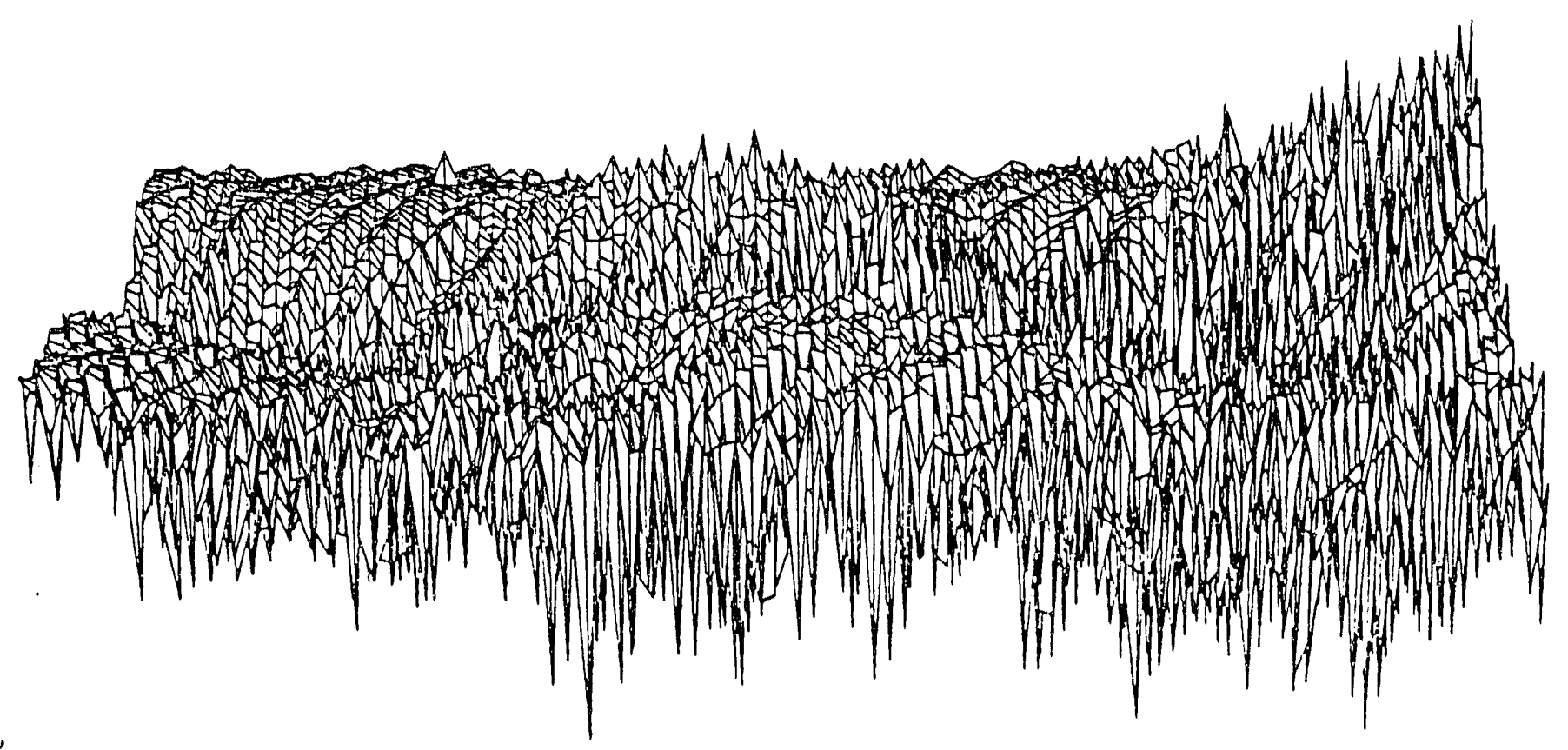




\section{Field Distribution in a Distributed Feed Back Laser}

Semiconductor lasers provide a useful low power source into a fiber optics communications channel. High mode selectivity is needed to obtain propagation of a single mode down a fiber. A DFB structure was proposed as a good candidate for a single mode semiconductor laser.

The rate at which the information can be transmitted is limited by noise. The principal noise comes from the popping up a non-lasing mode. Even though that the total power is not effected by this kind of noise, the new mode is at a different frequency and propagates with a different velocity, therefore causing the bit of information to arrive at an incorrect time.

Based on the solution of the mode equations with a uniform gain distribution the estimates were made of the gain differential between two modes to keep the bit error rate produced by partition fluctuations below a prescribed amount. Because nonuniform hole burning occurs, leading to a nonuniform gain, it is imperative to deal with the spatial distribution of carriers and field, and not just the total number of photons and carriers.

We have solved the stationary nonuniform mode equations and obtained the main mode profile for a DFB lasers. We have also obtained the gain differential between the main and the side modes of the DFB laser.

${ }^{1}$ W. Cai, T. F. Zheng, P. Hu, B. Yudanin, and M. Lax, "Model of phonon-associated electron tunneling through a semiconductor double barrier' Phys. Rev. Lett. 63, 418 (1989).

${ }^{2}$ W. Cai, P. Hu, T. F. Zheng, B. Yudanin, and M. Lax, "Resonance of the one-dimensional electron transmission above a quantum well with dissipation" Phys. Rev. B 41, 3513 (1990).

${ }^{3}$ W. Cai, T. F. Zheng, P. Hu, M. Lax, K. Shum and R. R. Alfano, "Photon-assisted resonant tunneling through a double-barrier structure for infrared-radiation detection" Phys. Rev. Lett. 65, 104 (1990).

${ }^{4}$ W. Cai, P. Hu, and M. Lax, "Wave behavior of high-frequency current through a doublebarrier tunneling structure", Phys. Rev. B 44, 3336 (1991).

${ }^{5}$ R. P. Feynman and F. L. Vernon, Ann. Phys. (N.Y.) 24, 118 (1963).

${ }^{6}$ P. Hu, W. Cai, T.F. Zheng, and M. Lax "Electron tunneling with dissipation", submitted to Phys. Rev B. 
${ }^{7}$ W. Cai, P. Hu, T. F. Zheng, B. Yudanin, and M. Lax, "Nonlinear high-frequency conductivity in semiconductors"' Phys. Rev. B 40, 7671 (1989).

${ }^{8}$ T. F. Zheng, W. Cai, P. Hu, M. Lax, Kai Shum, and R. R. Alfano, "Resonant level lifetime in GaAs-AlAs double-barrier structure including $\Gamma-X$ mixing', in J. Appl. Phys. 69, 8387-8391, (1991).

${ }^{9}$ V. F. Turchin, V. P. Koslov and M. S. Malkevich, "The Use of Mathematical-Statistics Methods in the Solution of of Incorrectly Posed Problems," Sov. Physics, USPEKHI, 13, 681-840, (1971). [ 102, Nos. 3 and 4].

10 Joel N. Franklin, "Well-Posed Stochastic Extensions of Ill-Posed Linear Problems," J. Math. Analys. and Applic. 31, 682-716 (1970).

$11 \mathrm{~J}$. Neyman and E. S. Pearson, "On the Problem of the Most Efficient Tests of Statistical Hypotheses," Philosophical Trans. A, 231, 289 (1933)

${ }^{12}$ M. G. Kendall and A. Stuart, The Advanced Theory of Statistics, Volume 2 Hafner Publishing Co, New York (1967)

${ }^{13}$ H. L. Van Trees, Detection, Estimation, and Modulation Theory, Part I, John Wiley and Sons, (1968)

${ }^{14}$ David Middleton, Introduction to Statistical Communication Theory, McGraw-Hill (1960) 


\section{PUBLICATIONS by M. LAX 1989.1992}

${ }^{1}$ T. F. Zheng, W. Cai, P. Hu and M. Lax, "Quasi-Analytical Simulation of Ultrafast Thermalization of Photoexcited Electrons in a semiconductor quantum well" Phys. Rev. B. 40, 1271 (1989).

${ }^{2}$ W. Cai, P. Hu, T. F. Zheng, B. Yudanin, and M. Lax, "Nonlinear high frequency conductivity in semiconductors" Phys. Rev. B. 40, (1989)

${ }^{3}$ W. Cai, P. Hu, T. F. Zheng, B. Yudanin, and M. Lax, "Electron transport in semiconductors under a strong high frequency electric field" Proceedings of 6th International Conference on Hot Carriers in Semiconductors, Solid-State Electronics 10\%7-1081, (1989).

${ }^{4}$ T. F. Zheng, W. Cai, P. Hu and M. Lax, "Simulation of ultrafast relaxation of photoexcited electrons via analytical distribution functions" Proceedings of 6th International Conference on Hot Carriers in Semiconductors, Solid-State Electronics 1089-1093, (1989).

${ }^{5}$ W. Cai. T. F. Zheng, P. Hu, B. Yudanin and M. Lax. "Model of PhononAssaciated Electron tumneling Through a Semiconductor Double Barrier" Phys. Rev. Letts. 63, 418, (1989)

${ }^{6}$ W. Cai, P. Hu, T. F. Zheng, B. Yudanin, and M. Lax. "Resonance of the One-dimensional Electron Transmission above a Quantum Well with Dissipation"' Phys. Rev. B 41, 3513-3516, (1990).

${ }^{7}$ M. Lax, W. Cai, P. Hu. T. F. Zheng, B. Yudanin and M. C. Marchetti "Coupling between 2-D Electrons in Quantum Wells and 3-D Phonons" in Frontiers in Condensed Matter Theory, Annals of the New York Academy of Sciences 581, American Institute of Physics Conference Proceedings 213, 195-206, (1990)

${ }^{8}$ W. Cai, T. F. Zheng, P. Hu, M. Lax, Kai Shum and R. R. Alfano "Photonassisted resonant tunneling through a double barrier structure for infrared 
radiation detection' Phys. Rev. Lett. 65, 104, (1990).

${ }^{9} \mathrm{Y}$. Q. Jin and M. Lax "Back-Scattering Enhancement From a Randomly Rough Surface" Phys. Rev. B 42, 9819-9829, (1990)

${ }^{10} \mathrm{~B}$. Yudanin and M. Lax, "POST Adaptation for a Numerical Solution of the Spherically-Symmetric Riemann Problem" in Int. Jour. of Mod. Phys. C, 1, 285-298, (1990)

${ }^{11}$ M. Lax, "The Theory of Laser Noise" Keynote address - 1990 Conference on Laser Science and Optics Applications - Boston, MA Proceedings SPIE 1376, 2-20 (1991)

${ }^{12} \mathrm{~W}$. Cai, T, F. Zheng, P. Hu, and M. Lax, "One-Dimensional Electron Tunneling in Semiconductors Including Inelastic Scattering" Mod. Phys. Letts. B 173-180, (1991)

${ }^{13}$ T. F. Zheng, W. Cai, P. Hu, M. Lax, Kai Shum, and R. R. Alfano, "Resonant level lifetime in GaAs-AlAs double-barrier structure including $\Gamma-\mathrm{X}$ mixing", in J. Appl. Phys. 69, 8387-8391, (1991)

${ }^{14}$ W. Cai, P. Hu, and M. Lax, "Wave behavior of high-frequency current through a double-barrier tunneling structure", Phys. Rev. B 44, 3336-3339, (1991)

${ }^{15}$ P. Hu and M. Lax, "Single Scattering Inversion Problem", Proceedings of the 1990 Scientific Conference on Obscuration and Aerosol Research, 87-98, (1991)

${ }^{16} \mathrm{~W}$. Cai and M. Lax, "Nonlinear Transport of Electrons Under a Strong High Frequency Electric Field in Semiconductors", Intl. Journal of Mod. Phys. 6 , 1007-1036, (1992)

${ }^{17}$ M. Lax and W. Cai, "Effect of Nonequilibrium Phonons on the Electron Relaxation and Transport", Intl. Journal of Mod. Phys. 6, 975-1006, (1992) 

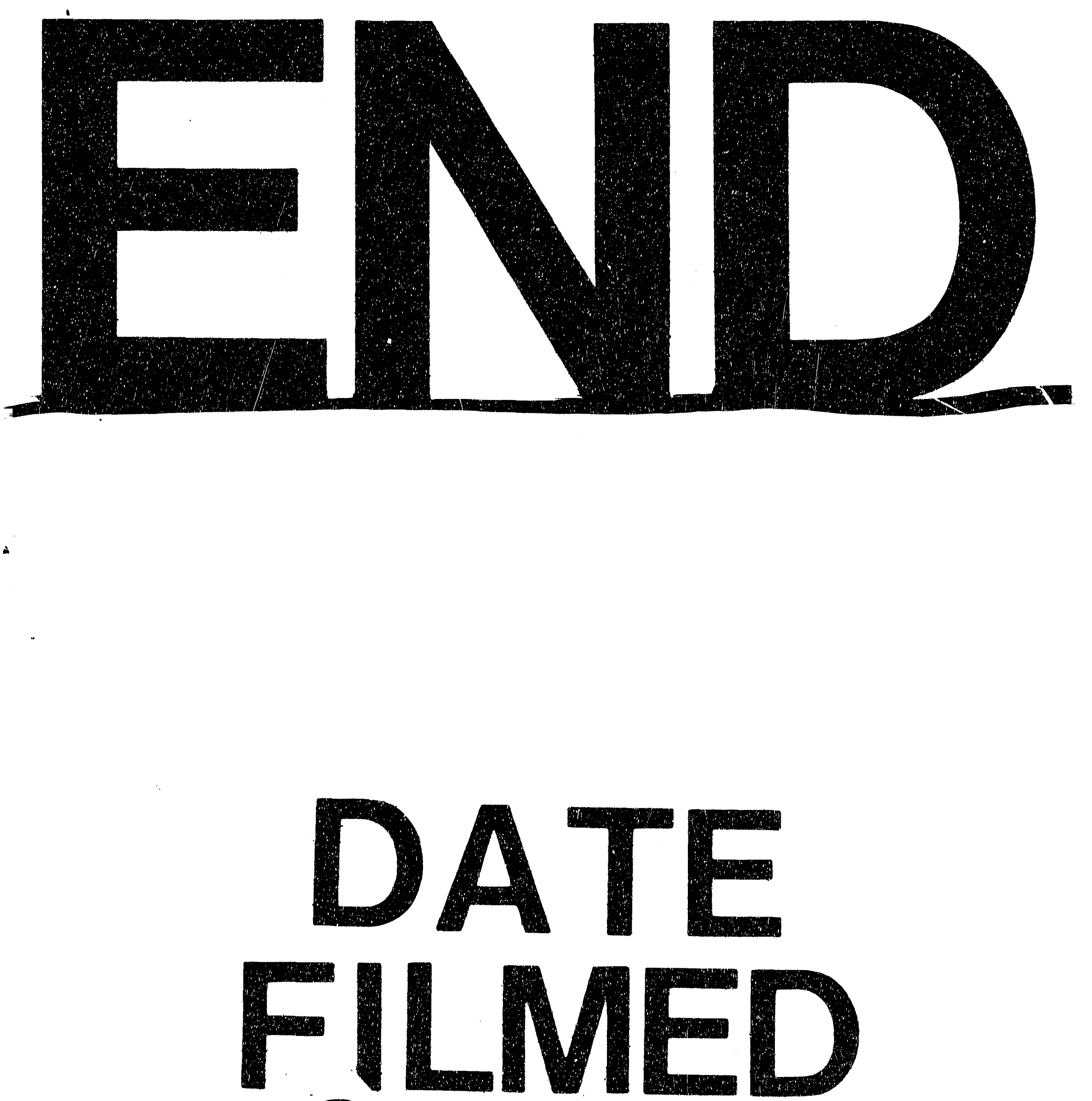
